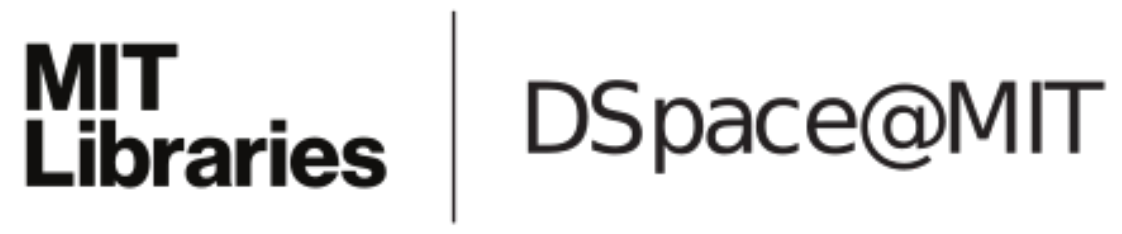

\author{
MIT Open Access Articles
}

Homomorphisms of Trees into a Path

The MIT Faculty has made this article openly available. Please share how this access benefits you. Your story matters.

Citation: Csikvari, Peter, and Zhicong Lin. "Homomorphisms of Trees into a Path." SIAM Journal on Discrete Mathematics 29, no. 3 (January 2015): 1406-1422. (c) 2015, Society for Industrial and Applied Mathematics

As Published: http://dx.doi.org/10.1137/140993995

Publisher: Society for Industrial and Applied Mathematics

Persistent URL: http://hdl.handle.net/1721.1/100547

Version: Final published version: final published article, as it appeared in a journal, conference proceedings, or other formally published context

Terms of Use: Article is made available in accordance with the publisher's policy and may be subject to US copyright law. Please refer to the publisher's site for terms of use. 


\title{
HOMOMORPHISMS OF TREES INTO A PATH*
}

\author{
PÉTER CSIKVÁRI ${ }^{\dagger}$ AND ZHICONG LIN ${ }^{\ddagger}$
}

\begin{abstract}
Let $\operatorname{hom}(G, H)$ denote the number of homomorphisms from a graph $G$ to a graph $H$. In this paper we study the number of homomorphisms of trees into a path, and prove that $\operatorname{hom}\left(P_{m}, P_{n}\right) \leq \operatorname{hom}\left(T_{m}, P_{n}\right) \leq \operatorname{hom}\left(S_{m}, P_{n}\right)$, where $T_{m}$ is any tree on $m$ vertices, and $P_{m}$ and $S_{m}$ denote the path and star on $m$ vertices, respectively. This completes the study of extremal problems concerning the number of homomorphisms between trees started in the paper Graph Homomorphisms Between Trees [Electron. J. Combin., 21 (2014), 4.9] written by the authors of the current paper.
\end{abstract}

Key words. homomorphisms, adjacency matrix, extremal problems, KC-transformation, path, star

AMS subject classifications. 05C05, 05C30, 05C35

DOI. $10.1137 / 140993995$

1. Introduction. There has been recent interest in enumerative and extremal problems related to graph homomorphisms $[4,8,9,10]$. This paper is the second part of our systematic study of extremal problems on counting graph homomorphisms between trees. For the first part, see [7].

All the graphs considered here are finite and undirected without multiple edges and loops. Recall that a homomorphism from a graph $G$ to a graph $H$ is a mapping $f$ : $V(G) \rightarrow V(H)$ such that the images of adjacent vertices are adjacent. Let $\operatorname{Hom}(G, H)$ be the set of homomorphisms from $G$ to $H$ and $\operatorname{hom}(G, H):=|\operatorname{Hom}(G, H)|$. Throughout this article, we write $P_{n}$ and $S_{n}$ for the path and the star on $n$ vertices, respectively.

In the paper [7], the authors started to study the extremal number of homomorphisms between trees. The current paper and paper [7] together give the following relations between homomorphism numbers summarized in Figures 1 and 2 (the " $X$ " means that there is no inequality between the two expressions in general and the "?" means that we do not know whether the statement is true or not).

In the paper [7] we studied mainly, but not exclusively, inequalities of type

$$
\operatorname{hom}\left(T_{m}, T\right) \leq \operatorname{hom}\left(T_{m}, T^{\prime}\right)
$$

In other words, we mainly studied the row inequalities. In the current paper, we study the first columns in both tables and prove the corresponding inequalities. The main difference between the current paper and [7] is in the methods. In [7] we developed quite general methods to examine the number of homomorphisms between graphs. Those methods were sufficient to give good results, but they never provided very precise ones. In the current paper, we study only homomorphisms of trees into paths,

\footnotetext{
* Received by the editors November 3, 2014; accepted for publication (in revised form) May 22, 2015; published electronically August 6, 2015.

http://www.siam.org/journals/sidma/29-3/99399.html

${ }^{\dagger}$ Department of Mathematics, Massachusetts Institute of Technology, Cambridge MA 02139 and Department of Computer Science, Eötvös Loránd University, H-1117 Budapest, Pázmány Péter sétány 1/C, Hungary (peter.csikvari@gmail.com). This author is partially supported by MTA Rényi "Lendület" Groups and Graphs Research Group and by the National Science Foundation by grant DMS-1500219.

${ }_{\ddagger}^{\ddagger}$ School of Sciences, Jimei University, Xiamen 361021, People’s Republic of China and CAMP, National Institute for Mathematical Sciences, Daejeon 305-811, Republic of Korea (lin@math.univlyon1.fr).
} 


$$
\begin{aligned}
& \operatorname{hom}\left(P_{m}, P_{n}\right) \leq \operatorname{hom}\left(P_{m}, T_{n}\right) \leq \operatorname{hom}\left(P_{m}, S_{n}\right) \\
& \wedge \text { I } \quad X \quad \wedge \\
& \operatorname{hom}\left(T_{m}, P_{n}\right) \stackrel{(*)}{\leq} \operatorname{hom}\left(T_{m}, T_{n}\right) X \operatorname{hom}\left(T_{m}, S_{n}\right) \\
& \wedge \text { । } \wedge \text { । } \wedge \\
& \operatorname{hom}\left(S_{m}, P_{n}\right) \leq \operatorname{hom}\left(S_{m}, T_{n}\right) \leq \operatorname{hom}\left(S_{m}, S_{n}\right)
\end{aligned}
$$

FIG. 1. The number of homomorphisms between trees of different sizes. The $(*)$ means that there are some well-determined (possible) counterexamples which should be excluded.

$$
\begin{array}{ccc}
\operatorname{hom}\left(P_{n}, P_{n}\right) & \leq \operatorname{hom}\left(P_{n}, T_{n}\right) & \leq \operatorname{hom}\left(P_{n}, S_{n}\right) \\
\wedge \mathrm{l} & ? & \wedge \mathrm{l} \\
\operatorname{hom}\left(T_{n}, P_{n}\right) & \leq \operatorname{hom}\left(T_{n}, T_{n}\right) & X \operatorname{hom}\left(T_{n}, S_{n}\right) \\
\wedge \mathrm{I} & \wedge \mathrm{l} & \wedge \mathrm{l} \\
\operatorname{hom}\left(S_{n}, P_{n}\right) & \leq \operatorname{hom}\left(S_{n}, T_{n}\right) & \leq \operatorname{hom}\left(S_{n}, S_{n}\right)
\end{array}
$$

FIG. 2. The number of homomorphisms between trees of the same size.

but the problem enables us to use much more subtle arguments which are unavailable in general for arbitrary trees.

In $[5,6]$, a graph transformation defined on trees called $K C$-transformation (see section 2.2 for its definition) or generalized tree shift was shown to be a powerful tool in proving that a certain graph parameter attains its maximum at the star $S_{n}$ and its minimum at the path $P_{n}$ among the trees on $n$ vertices. Furthermore, Bollobás and Tyomkyn [2] showed that the KC-transformation increases the number of walks of fixed length on trees, which implies the extremal property

$$
\operatorname{hom}\left(P_{m}, P_{n}\right) \leq \operatorname{hom}\left(P_{m}, T_{n}\right) \leq \operatorname{hom}\left(P_{m}, S_{n}\right),
$$

where $T_{n}$ is any tree on $n$ vertices. Note that inequality (1.1) was generalized in [7] from $P_{m}$ to trees with at most one vertex of degree greater than two. The main result of this paper is the following dual of inequality (1.1), concerning the number of homomorphisms of a tree into a path.

Theorem 1.1. Let $T_{m}$ be a tree on $m$ vertices. Furthermore, let $\operatorname{diam}\left(T_{m}\right)$ denote the diameter of $T_{m}$.

(i) Let $T_{m}^{\prime}$ be obtained from $T_{m}$ by a KC-transformation. If $n$ is even, or $n$ is odd and $\operatorname{diam}\left(T_{m}\right) \leq n-1$, then

$$
\operatorname{hom}\left(T_{m}, P_{n}\right) \leq \operatorname{hom}\left(T_{m}^{\prime}, P_{n}\right) \text {. }
$$

(ii) For any $m, n$,

$$
\operatorname{hom}\left(P_{m}, P_{n}\right) \leq \operatorname{hom}\left(T_{m}, P_{n}\right) \leq \operatorname{hom}\left(S_{m}, P_{n}\right) .
$$

Remark 1.2. The inequality (1.2) is not true in general when $n$ is odd and $\operatorname{diam}\left(T_{m}\right)$ is greater than $n-1$; see a counterexample in Figure 3 . The first inequality of (1.3) is an important step in the proof of [7, Theorem 1.8], and its proof needs more tree transformations. The second inequality of (1.3) is a special case of a result due to Sidorenko [11]:

$$
\operatorname{hom}\left(T_{m}, G\right) \leq \operatorname{hom}\left(S_{m}, G\right)
$$



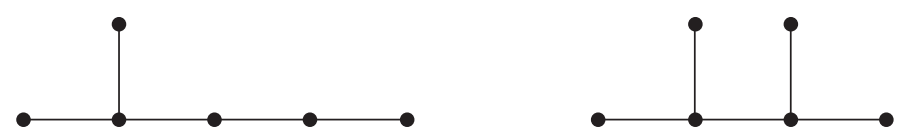

FIG. 3. The trees $T_{6}$ (left) and $T_{6}^{\prime}$ (right).

for any graph $G$. See also [7] for a neat proof of Sidorenko's result using the Wiener index of trees.

The rest of this paper is devoted to proving Theorem 1.1. As Theorem 1.1 suggests the cases of $n$ being even or odd are somewhat different. We treat part (i) of Theorem 1.1 and the case of even $n$ of part (ii) in section 3, while we treat the case of odd $n$ of part (ii) in section 4. Let us mention that in the case of odd $n$ we will also distinguish two cases according to $m$ being even or odd. This paper is concluded with some interesting open questions. The reader is referred to $[1,3]$ for any terminology not defined herein.

\section{Preliminaries.}

2.1. Tree-walk algorithm. We will give an outline of the tree-walk algorithm developed in [7] to compute the number of homomorphisms from trees.

Let $\mathbf{a}=\left(a_{1}, a_{2}, \ldots, a_{n}\right)$ and $\mathbf{b}=\left(b_{1}, b_{2}, \ldots, b_{n}\right)$ be two vectors. We usually denote by $\|\mathbf{a}\|_{1}=a_{1}+a_{2}+\cdots+a_{n}$ the norm of $\mathbf{a}$ and by $\mathbf{a} * \mathbf{b}=\left(a_{1} b_{1}, \ldots, a_{n} b_{n}\right)$ the Hadamard product of $\mathbf{a}$ and $\mathbf{b}$. Let $G$ be a graph with $n$ vertices. The adjacency matrix of $G$ is the $n \times n$ matrix $A_{G}:=\left(a_{u v}\right)_{u, v \in V(G)}$, where $a_{u v}=1$ when $u v \in E(G)$, otherwise 0 .

DEFINITION 2.1 (hom-vector). Let $T$ be a tree and $G$ be a graph with vertices labeled by $1,2, \ldots, n$. Let $v \in V(T)$ be any vertex of $T$. The $n$-dimensional vector

$$
\mathbf{h}(T, v, G):=\left(h_{1}, h_{2}, \ldots, h_{n}\right),
$$

where

$$
h_{i}=|\{f \in \operatorname{Hom}(T, G) \mid f(v)=i\}|,
$$

is called the hom-vector at $v$ from $T$ to $G$ or the hom-vector from the rooted tree $T(v)$ to $G$. Clearly, $\operatorname{hom}(T, G)=\|\mathbf{h}(T, v, G)\|_{1}$.

The tree-walk algorithm comprises the following two type of recursion steps.

Recursion 1. If we have a tree $T$ with a nonleaf root vertex $v$, then we can decompose $T$ to $T_{1} \cup T_{2}$ such that $V\left(T_{1}\right) \cap V\left(T_{2}\right)=\{v\}$, and $T_{1}$ and $T_{2}$ are strictly smaller than $T$. In this case

$$
\mathbf{h}(T, v, G)=\mathbf{h}\left(T_{1}, v, G\right) * \mathbf{h}\left(T_{2}, v, G\right) .
$$

Recursion 2. If we have a tree $T$ with a root vertex $v$ which is a leaf with the unique neighbor $u$, then

$$
\mathbf{h}(T, v, G)=\mathbf{h}(T-v, u, G) A,
$$

where $A$ is the adjacency matrix of $G$.

2.2. KC-transformation on trees. The KC-transformation on trees was first introduced in [5] under the name generalized tree shift. The reader can find many applications of this transformation in $[2,5,6,7]$. 

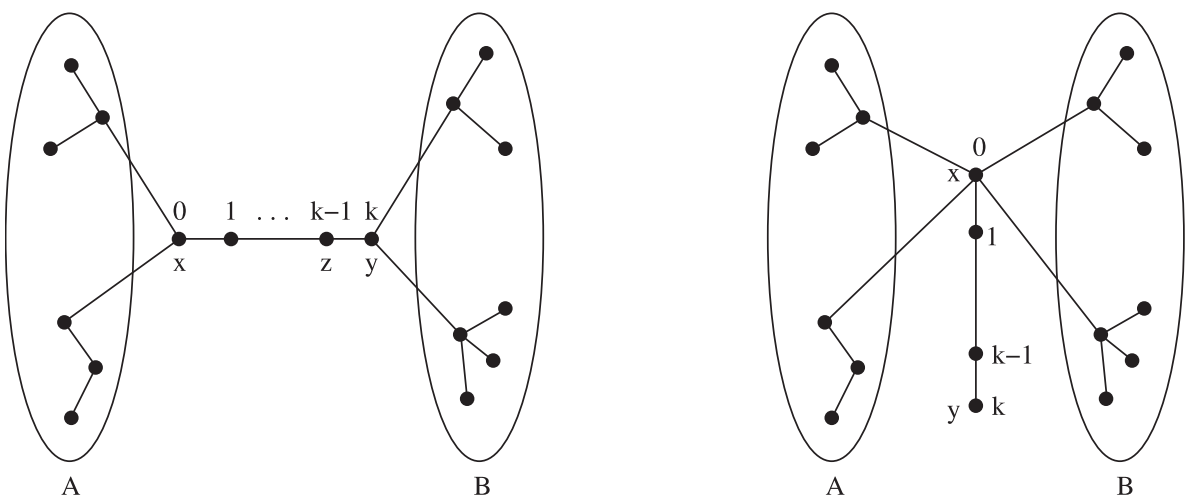

FIG. 4. The KC-transformation.

To define this transformation, let $x$ and $y$ be two vertices of a tree $T$ such that every interior vertex of the unique $x-y$ path $P$ in $T$ has degree two, and write $z$ for the neighbor of $y$ on this path. Denote by $N(v)$ the set of neighbors of a vertex $v$. The KC-transformation, $K C(T, x, y)$, of the tree $T$ with respect to the path $P$ is obtained from $T$ by deleting all edges between $y$ and $N(y) \backslash z$ and adding the edges between $x$ and $N(y) \backslash z$ instead (see Figure 4). Note that $K C(T, x, y)$ and $K C(T, y, x)$ are isomorphic.

The following property of KC-transformation was proved in [5].

Proposition 2.2. The KC-transformation gives rise to a graded poset of trees on $n$ vertices (graded by the number of leaves) with the star $S_{n}$ as the largest and the path $P_{n}$ as the smallest element.

3. The KC-transformation: Proof of Theorem 1.1(i). In this section we will prove part (i) of Theorem 1.1 by making use of the KC-transformation on trees, and we settle the case of even $n$ in part (ii) of Theorem 1.1. First we need some new definitions and lemmas.

Definition 3.1. A vector $\mathbf{a}=\left(a_{1}, a_{2}, \ldots, a_{n}\right)$ is symmetric if $a_{i}=a_{n-i+1}$ for $1 \leq i \leq n-1$, and unimodal if $a_{1} \leq a_{2} \leq \cdots \leq a_{j} \geq a_{j+1} \geq \cdots \geq a_{n}$ for some $j$. Denote by $R_{n}$ the set of symmetric positive integer vectors of dimension $n$. For any $\mathbf{a}, \mathbf{b} \in R_{n}$, define the dominance order on $R_{n}$ by

$$
\mathbf{a} \preceq \mathbf{b} \Leftrightarrow \sum_{i=k}^{n+1-k} a_{i} \leq \sum_{i=k}^{n+1-k} b_{i} \quad \text { for } \quad 1 \leq k \leq\lceil n / 2\rceil .
$$

It is clear that $R_{n}$ is a poset with respect to this order and $\mathbf{a} \preceq \mathbf{b}$ implies $\|\mathbf{a}\|_{1} \leq\|\mathbf{b}\|_{1}$. Let $U_{n}$ be the set of all unimodal vectors in $R_{n}$.

Lemma 3.2. Let $\mathbf{c} \in U_{n}$ and $\mathbf{a}, \mathbf{b} \in R_{n}$ such that $\mathbf{a} \preceq \mathbf{b}$. Then $\mathbf{a} * \mathbf{c} \preceq \mathbf{b} * \mathbf{c}$.

Proof. It is clear that both $\mathbf{a} * \mathbf{c}$ and $\mathbf{b} * \mathbf{c}$ are in $R_{n}$. For $1 \leq k \leq\left\lceil\frac{n}{2}\right\rceil$, by the symmetric property of $\mathbf{a}, \mathbf{b}$, and $\mathbf{c}$, we have the identity

$$
\sum_{i=k}^{n+1-k}\left(b_{i}-a_{i}\right) c_{i}=c_{k-1} \sum_{i=k}^{n+1-k}\left(b_{i}-a_{i}\right)+\sum_{j=k}^{\left\lceil\frac{n}{2}\right\rceil}\left(c_{j}-c_{j-1}\right) \sum_{i=j}^{n+1-j}\left(b_{i}-a_{i}\right),
$$

where $c_{0}=0$. From $\mathbf{c} \in U_{n}$ and $\mathbf{a} \preceq \mathbf{b}$, we see that $c_{j}-c_{j-1} \geq 0$ and $\sum_{i=j}^{n+1-j}\left(b_{i}-a_{i}\right) \geq$ 0 for $1 \leq j \leq\left\lceil\frac{n}{2}\right\rceil$. Thus the right-hand side of the above identity is nonnegative, which is equivalent to $\mathbf{a} * \mathbf{c} \preceq \mathbf{b} * \mathbf{c}$. 
It is clear that if $a \in U_{2 n}$, then $a A^{k} \in U_{2 n}$ for any positive integer $k$, where $A$ is the adjacency matrix of $P_{2 n}$. But for the path with odd vertices, this is not true in general. Let $\mathbf{1}_{\mathbf{n}}$ be the $n$-dimensional row vector with all entries equal to 1 .

Lemma 3.3. Let $A$ be the adjacency matrix of $P_{n}$ and $l$ a positive integer. If $\mathbf{a} \in U_{n}$, then $\mathbf{a} A^{l} \preceq \mathbf{a} *\left(\mathbf{1}_{\mathbf{n}} A^{l}\right)$.

Proof. Let $\mathbf{a}=\left(a_{1}, \ldots, a_{n}\right), \mathbf{a} A^{l}=\left(x_{1}, \ldots, x_{n}\right)$, and $\mathbf{a} *\left(\mathbf{1}_{\mathbf{n}} A^{l}\right)=\left(y_{1}, y_{2}, \ldots, y_{n}\right)$. For $1 \leq j \leq n$, the sum of coefficients of all $a_{i}(1 \leq i \leq n)$ in $x_{j}$ is the sum of the $j$ th column of $A^{l}$ and the coefficient of $a_{j}$ in $y_{j}$ also equals the sum of the $j$ th column of $A^{l}$. It follows that the sum of coefficients of all $a_{i}(1 \leq i \leq n)$ in $\sum_{i=k}^{n+1-k} x_{i}$ equals the sum of coefficients of all $a_{i}(1 \leq i \leq n)$ in $\sum_{i=k}^{n+1-k} y_{i}$. But for every $i$, the coefficient of $a_{i}$ in $\left\|\mathbf{a} A^{l}\right\|_{1}$ is the sum of $i$ th row of $A^{l}$ and the coefficient of $a_{i}$ in $\left\|\mathbf{a} * \mathbf{1}_{\mathbf{n}} A^{l}\right\|_{1}$ is the sum of $i$ th column of $A^{l}$, which are equal. Thus $\sum_{i=k}^{n+1-k} x_{i} \leq \sum_{i=k}^{n+1-k} y_{i}$ follows from the unimodality of $\mathbf{a}$, which shows that $\mathbf{a} A^{l} \preceq \mathbf{a} *\left(\mathbf{1}_{\mathbf{n}} A^{l}\right)$.

Before we prove part (i) of Theorem 1.1, we show that we only have to prove the statement for the case of even $n$ since the following lemma implies it for the case of odd $n$ if $\operatorname{diam}(T) \leq n-1$.

Lemma 3.4. If $\operatorname{diam}(T) \leq n-1$, then

$$
\operatorname{hom}\left(T, P_{n}\right)=\frac{1}{2}\left(\operatorname{hom}\left(T, P_{n-1}\right)+\operatorname{hom}\left(T, P_{n+1}\right)\right) .
$$

Proof. Let the vertices of $P_{n}$ be labeled consecutively by $1,2, \ldots, n$. We can decompose the set $\operatorname{Hom}\left(T, P_{n}\right)$ to the following two sets. The first set consists of those homomorphisms which do not contain the vertex $n$ in their image and the second set consists of those homomorphisms which contain the vertex $n$ in their image. Clearly, the cardinality of the first set is $\operatorname{hom}\left(T, P_{n-1}\right)$. The cardinality of the second set will be denoted by $\operatorname{hom}\left(T, P_{n}, n \in f(T)\right)$. So

$$
\operatorname{hom}\left(T, P_{n}\right)=\operatorname{hom}\left(T, P_{n-1}\right)+\operatorname{hom}\left(T, P_{n}, n \in f(T)\right) .
$$

We can repeat this argument with the path $P_{n+1}$ as well:

$$
\operatorname{hom}\left(T, P_{n+1}\right)=\operatorname{hom}\left(T, P_{n}\right)+\operatorname{hom}\left(T, P_{n+1}, n+1 \in f(T)\right) .
$$

Rearranging this we obtain that

$$
\operatorname{hom}\left(T, P_{n}\right)=\operatorname{hom}\left(T, P_{n+1}\right)-\operatorname{hom}\left(T, P_{n+1}, n+1 \in f(T)\right) .
$$

The crucial observation is that

$$
\operatorname{hom}\left(T, P_{n}, n \in f(T)\right)=\operatorname{hom}\left(T, P_{n+1}, n+1 \in f(T)\right) .
$$

Indeed, if $n+1 \in f(T)$, then $1 \notin f(T)$ because $\operatorname{diam}(T)<\operatorname{diam}\left(P_{n+1}\right)$, so all these homomorphisms go to the path $\{2,3, \ldots, n+1\}$ and therefore there is a natural correspondence between the two sets. Hence if $\operatorname{diam}(T) \leq \operatorname{diam}\left(P_{n}\right)$, then by adding together (3.2) and (3.3) we obtain (3.1).

Proof of Theorem 1.1(i). Let $A$ be the adjacency matrix of $P_{n}$. Let $T_{m}^{\prime}=$ $K C(T, x, y)$ be the KC-transformation of the tree $T_{m}$ with respect to an $x-y$ path $P$. Let $B_{1}$ and $B_{2}$ be the components of $y$ and $x$ in the subgraph of $T_{m}$ by deleting all the edges of $P$, respectively.

Consider first the case where $n$ is even. It is easy to see that an element of $U_{n}$ multiplied by $A$ is still in $U_{n}$ and the Hadamard product of two elements in $U_{n}$ is also 
in $U_{n}$. By the tree-walk algorithm, any hom-vector from a tree to $P_{n}$ is in $U_{n}$. Again by the tree-walk algorithm, we have

$$
\mathbf{h}\left(T_{m}, x, P_{n}\right)=\left(\mathbf{h}\left(B_{1}, y, P_{n}\right) A^{k}\right) * \mathbf{h}\left(B_{2}, x, P_{n}\right)
$$

and

$$
\mathbf{h}\left(T_{m}^{\prime}, x, P_{n}\right)=\mathbf{h}\left(B_{1}, y, P_{n}\right) * \mathbf{1}_{\mathbf{n}} A^{k} * \mathbf{h}\left(B_{2}, x, P_{n}\right),
$$

where $k$ is the length of the path $P$. By Lemma 3.3, we have

$$
\mathbf{h}\left(B_{1}, y, P_{n}\right) A^{k} \preceq \mathbf{h}\left(B_{1}, y, P_{n}\right) * \mathbf{1}_{\mathbf{n}} A^{k} .
$$

It follows from Lemma 3.2 that $\mathbf{h}\left(T, x, P_{n}\right) \preceq \mathbf{h}\left(T^{\prime}, x, P_{n}\right)$, which implies $\operatorname{hom}\left(T, P_{n}\right) \leq$ $\operatorname{hom}\left(T^{\prime}, P_{n}\right)$.

For the case of odd $n$ we have already seen that Lemma 3.4 implies the statement.

Theorem 1.1(ii): $n$ is even. Let $n$ be even. Then for any tree $T_{m}$ on $m$ vertices we have

$$
\operatorname{hom}\left(T_{m}, P_{n}\right) \geq \operatorname{hom}\left(P_{m}, P_{n}\right) .
$$

Proof. This statement immediately follows from part (i) of Theorem 1.1 and Proposition 2.2.

Remark 3.5. The KC-transformation does not always increase the number of homomorphisms to the path $P_{n}$ when $n$ is odd. For example, in Figure 3, we have $\operatorname{hom}\left(T_{6}, P_{3}\right)=20>16=\operatorname{hom}\left(T_{6}^{\prime}, P_{3}\right)$.

4. More tree transformations: Case of odd $n$ in Theorem 1.1(ii). By Proposition 2.2, if $n$ is even or $n$ is odd and $\operatorname{diam}\left(T_{m}\right) \leq n-1$, then Theorem 1.1(i) implies part (ii). In this section, we will develop some more transformations on trees to prove Theorem 1.1(ii) for odd $n$. So from now on in this section, $n$ is odd.

For any $u \in V(T)$, denote by $T(u)$ the rooted tree with a root at $u$. As usual, we will denote by $\mathbf{h}(T, u, G)$ the hom-vector of the rooted tree $T(u)$ into $G$. One can easily check that the hom-vector of a rooted tree into $P_{n}$, i.e., $\mathbf{h}\left(T, u, P_{n}\right)$, is not unimodal anymore. On the other hand, the situation is not as bad as one may think at first sight.

DEFINITION 4.1 (symmetric and biunimodal). We say that $\left(a_{1}, a_{2}, \ldots, a_{n}\right)$ is symmetric biunimodal if the sequence itself is symmetric and the two subsequences $\left(a_{1}, a_{3}, \ldots, a_{n}\right)$ and $\left(a_{2}, a_{4}, \ldots, a_{n-1}\right)$ are unimodal.

Proposition 4.2. Let $T(u)$ be a rooted tree and $\left(a_{1}, \ldots, a_{n}\right)$ be the hom-vector of $T(u)$ into $P_{n}$. Then $\left(a_{1}, \ldots, a_{n}\right)$ is symmetric biunimodal.

Proof. This statement follows from the tree-walk algorithm and the observation that if $\left(a_{1}, a_{2}, \ldots, a_{n}\right)$ and $\left(b_{1}, b_{2}, \ldots, b_{n}\right)$ are symmetric biunimodal, then the sequences $\left(a_{1} b_{1}, a_{2} b_{2}, \ldots, a_{n} b_{n}\right)$ and $\left(a_{2}, a_{1}+a_{3}, \ldots, a_{n-2}+a_{n}, a_{n-1}\right)$ are also symmetric biunimodal.

The following is a very surprising theorem. In fact, it is not true for even $n$ (see Remark 4.4).

LEMma 4.3 (correlation inequality). Let $T_{1}(u)$ be a rooted tree and $T_{2}(u)$ be a rooted subtree, i.e., $T_{2}$ is a subtree of $T_{1}$ and their roots are the same. Let $\left(a_{1}, \ldots, a_{n}\right)$ (resp., $\left(b_{1}, \ldots, b_{n}\right)$ ) be the hom-vector of $T_{1}(u)$ (resp., $\left.T_{2}(u)\right)$ into $P_{n}$, where $n$ is odd. 
Assume that $i \equiv j \quad(\bmod 2)$ and $\left|\frac{n+1}{2}-i\right| \leq\left|\frac{n+1}{2}-j\right|$, so $i$ is closer to the center than $j$. Then, $a_{i} b_{j} \geq a_{j} b_{i}$.

Proof. We need to prove that $\frac{a_{i}}{a_{j}} \geq \frac{b_{i}}{b_{j}}$. (From this form it is clear that if we introduce the function $f_{i j}=\frac{a_{i}}{a_{j}}$ for rooted trees, then we need to prove that if $T_{2}(u)$ is a rooted subtree of $T_{1}(u)$, then $f_{i j}\left(T_{1}(u)\right) \geq f_{i j}\left(T_{2}(u)\right)$.) We prove this claim by induction on the number of vertices of $T_{1}(u)$. If $\left|T_{1}(u)\right| \leq 2$, then it is trivial to check the statement.

If $u$ is not a leaf in $T_{1}$, then by induction this inequality holds for the branches at $u$ and then it is true for the Hadamard products. If $u$ is a leaf in $T_{1}$, then it is also a leaf in $T_{2}$. Let $v$ be the unique neighbor of $u$ and let us consider the rooted trees $\left(T_{1}-u\right)(v)$ and $\left(T_{2}-u\right)(v)$. Let $\left(a_{1}^{\prime}, a_{2}^{\prime}, \ldots, a_{n}^{\prime}\right)$ and $\left(b_{1}^{\prime}, \ldots, b_{n}^{\prime}\right)$ be their hom-vectors. Then,

$$
a_{i}=a_{i-1}^{\prime}+a_{i+1}^{\prime} \text { and } b_{i}=b_{i-1}^{\prime}+b_{i+1}^{\prime},
$$

where $a_{0}^{\prime}=a_{n+1}^{\prime}=b_{0}^{\prime}=b_{n+1}^{\prime}=0$. Note that the numbers $i-1, i+1, j-1, j+1$ are still congruent modulo 2 . Because of the symmetry we can assume that $j<i \leq \frac{n+1}{2}$. If, in addition, $i<\frac{n+1}{2}$, then we have $j-1<j+1 \leq i-1<i+1 \leq \frac{n+1}{2}$ and we can apply the induction hypothesis

$$
a_{i \pm 1}^{\prime} b_{j \pm 1}^{\prime} \geq a_{j \pm 1}^{\prime} b_{i \pm 1}^{\prime}
$$

in all four cases, thus,

$$
a_{i} b_{j}=\left(a_{i-1}^{\prime}+a_{i+1}^{\prime}\right)\left(b_{j-1}^{\prime}+b_{j+1}^{\prime}\right) \geq\left(a_{j-1}^{\prime}+a_{j+1}^{\prime}\right)\left(b_{i-1}^{\prime}+b_{i+1}^{\prime}\right)=a_{j} b_{i} .
$$

If $i=\frac{n+1}{2}$, then the above four inequalities are still true, because the $\{i-1, i+1\}$ are still closer to $\frac{n+1}{2}$ than the numbers $\{j-1, j+1\}$. Hence we have proved the statement.

Remark 4.4. As we mentioned Lemma 4.3 is not true for even $n$. Indeed, let $T_{1}(u)$ be $P_{3}$ with an end vertex being the root, and let $T_{2}(u)$ be $P_{2}$. Then $T_{2}(u)=P_{2}(u)$ is a rooted subtree of $T_{1}(u)$. Let us consider the homomorphisms of $T_{1}$ and $T_{2}$ going into $P_{4}$. The hom-vector of $T_{1}(u)$ is $(2,3,3,2)$, while the hom-vector of $T_{2}(u)$ is $(1,2,2,1)$. Now if we choose $i=3$ and $j=1$, then $\left|\frac{4+1}{2}-3\right| \leq\left|\frac{4+1}{2}-1\right|$, so 3 is closer to the center than 1 . On the other hand, we clearly have $3 \cdot 1<2 \cdot 2$ which shows that Lemma 4.3 is not true for even $n$. Similar examples exist for larger even $n$ 's.

Lemma 4.5 (log-concavity of the hom-vector.). Let $T_{1}(u)$ be a rooted tree and let $\left(a_{1}, \ldots, a_{n}\right)$ be the hom-vector of $T_{1}(u)$ into $P_{n}$, where $n$ is odd. Assume that $i<j$ and $i \not \equiv j(\bmod 2)$. Then $a_{i} a_{j} \leq a_{i+1} a_{j-1}$.

Proof. The proof is almost identical to the proof of the correlation inequality and thus is omitted.

Definition 4.6. Let $T_{1}, T_{2}$ be trees. Let $u$ be an arbitrary vertex of $T_{1}$ and let $A$ and $B$ be the color classes of $T_{2}$ considered as a bipartite graph. Let $h_{A}$ and $h_{B}$ be the number of homomorphisms from $T_{1}$ to $T_{2}$, where $u$ goes to $A$ and $B$, respectively. Then let

$$
g\left(T_{1}, T_{2}\right):=h_{A} h_{B}
$$

Note that $g\left(T_{1}, T_{2}\right)$ is independent of the vertex $u$. 
Let the vertices of $P_{n}$ be labeled consecutively by $1,2, \ldots, n$. If $T_{2}=P_{n}$, then $\operatorname{hom}_{0}\left(T_{1}(u), P_{n}\right)$ denotes the number of homomorphisms of $T_{1}$ into $P_{n}$, where the image of $u$ is a vertex of even index and $\operatorname{hom}_{1}\left(T_{1}(u), P_{n}\right)$ denotes the number of homomorphisms of $T_{1}$ into $P_{n}$, where the image of $u$ is a vertex of odd index. Thus in this case

$$
g\left(T_{1}, P_{n}\right)=\operatorname{hom}_{0}\left(T_{1}(u), P_{n}\right) \cdot \operatorname{hom}_{1}\left(T_{1}(u), P_{n}\right) .
$$

Remark 4.7. Before we proceed let us motivate the definition of the function g. Given two connected bipartite graphs $G_{1}=\left(A_{1}, B_{1}, E_{1}\right)$ and $G_{2}=\left(A_{2}, B_{2}, E_{2}\right)$, the set of homomorphisms from $G_{1}$ to $G_{2}$ naturally splits into two subsets according to whether the image of the color class $A_{1}$ is a subset of $A_{2}$ or $B_{2}$. It turns out that these two subsets can have very different cardinalities. Assume that $G_{2}$ is the complete bipartite graph $K_{r, s}=\left(A_{2}, B_{2}, E\right)$, where $\left|A_{2}\right|=r$ and $\left|B_{2}\right|=s$. Then the number of homomorphisms which maps $A_{1}$ to a subset of $A_{2}$ is $r^{\left|A_{1}\right|} s^{\left|B_{1}\right|}$ as any map which maps any vertex of $A_{1}$ to any element of $A_{2}$ and any vertex of $B_{1}$ to any element of $B_{2}$ is a proper homomorphism. Similarly, the number of homomorphisms which maps $A_{1}$ to a subset of $B_{2}$ is $r^{\left|B_{1}\right|} s^{\left|A_{1}\right|}$. If, say, $\left|A_{1}\right|$ and $r$ are large and $\left|B_{1}\right|$ and $s$ are small, then these two numbers have really different sizes. On the other hand, the function $g\left(G_{1}, G_{2}\right)=(r s)^{\left|A_{1}\right|+\left|B_{1}\right|}=(r s)^{\left|V\left(G_{1}\right)\right|}$ balances the two numbers nicely. In the particular case when $G_{1}$ is a tree $T$ and $r=2, s=1$, i.e., $G_{2}=P_{3}$, we get that $g\left(T, P_{3}\right)=2^{|T|}$.

The function $g\left(G_{1}, G_{2}\right)$ might look a bit artificial. The authors are not aware of the appearance of this function in the literature, nor is there a nice combinatorial meaning of this function. Nevertheless, this function seems to behave very nicely under tree transformations as, for instance, Theorem 4.8 shows.

We have the following crucial theorem on the function $g\left(T, P_{n}\right)$.

Theorem 4.8. Let $T_{m}$ be a tree on $m$ vertices. Then

$$
g\left(T_{m}, P_{n}\right) \geq g\left(P_{m}, P_{n}\right) .
$$

We will prove Theorem 4.8 by using two transformations: the LS-switch and the so-called short-path shift. The LS-switch was first introduced in [7] and is a generalization of the even-KC-transformation (i.e., $k$ is even in Figure 4 ).

Definition 4.9 (LS-switch). Let $R(u, v)$ be a tree with specified vertices $u$ and $v$ such that the distance of $u$ and $v$ is even and $R$ has an automorphism of order 2 which exchanges the vertices $u$ and $v$. Let $T_{1}(x), T_{2}(x), T_{3}(y), T_{4}(y)$ be rooted trees such that $T_{2}(x)$ is a rooted subtree of $T_{1}(x)$ and $T_{4}(y)$ is a rooted subtree of $T_{3}(y)$. Let the tree $T$ be obtained from the trees $R(u, v), T_{1}(x), T_{2}(x), T_{3}(y), T_{4}(y)$ by attaching a copy of $T_{1}(x), T_{4}(y)$ to $R(u, v)$ at vertex $u$ and a copy of $T_{2}(x), T_{3}(y)$ at vertex $v$. Suppose that the tree $T^{\prime}$ is obtained from the trees $R(u, v), T_{1}(x), T_{2}(x), T_{3}(y), T_{4}(y)$ by attaching a copy of $T_{1}(x), T_{3}(y)$ to $R(u, v)$ at vertex $u$ and a copy of $T_{2}(x), T_{4}(y)$ at vertex $v$ (see Figure 5). Then $T^{\prime}$ is called an LS-switch of $T$. Observe that there is a natural bijection between the color classes of $T^{\prime}$ and $T$.

This transformation seems to be quite general, but still the following result is true.

Lemma 4.10. Let $T$ be a tree and $T^{\prime}$ be an LS-switch of $T$. Then

$$
\operatorname{hom}_{k}\left(T^{\prime}(u), P_{n}\right) \geq \operatorname{hom}_{k}\left(T(u), P_{n}\right)
$$

for $k=0,1$. In particular,

$$
\operatorname{hom}\left(T^{\prime}, P_{n}\right) \geq \operatorname{hom}\left(T, P_{n}\right) \quad \text { and } \quad g\left(T^{\prime}, P_{n}\right) \geq g\left(T, P_{n}\right) .
$$



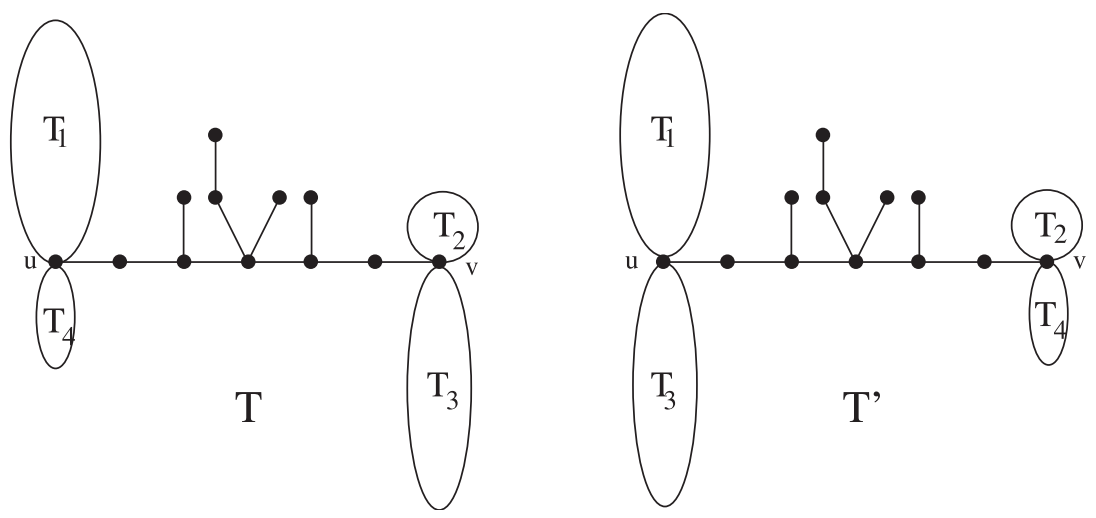

FIG. 5. LS-switch.

Proof. Let the vertices of $P_{n}$ be labeled consecutively by $1,2, \ldots, n$. For a rooted tree $T(r)$ let $h(T, i)$ denote the number of homomorphisms of $T$ into $P_{n}$ such that $r$ goes to the vertex $i$. So $(h(T, 1), h(T, 2), \ldots, h(T, n))$ is the hom-vector of $T(r)$ into $P_{n}$. Let $a_{i j}$ be the number of homomorphisms of $R(u, v)$ into $P_{n}$ such that $u$ goes to $i$ and $v$ goes to $j$. Note that $a_{i j}=a_{j i}$ because of the automorphism of order 2 of $R$ switching the vertices $u$ and $v$. Also note that $a_{i j}=0$ if $i$ and $j$ are incongruent modulo 2 since the distance of $u$ and $v$ is even. Observe that for $k=0,1$ we have

$$
\operatorname{hom}_{k}\left(T^{\prime}(u), P_{n}\right)=\sum_{i, j \equiv k(2)} a_{i j} h\left(T_{1}, i\right) h\left(T_{3}, i\right) h\left(T_{2}, j\right) h\left(T_{4}, j\right)
$$

and

$$
\operatorname{hom}_{k}\left(T(u), P_{n}\right)=\sum_{i, j \equiv k(2)} a_{i j} h\left(T_{1}, i\right) h\left(T_{4}, i\right) h\left(T_{2}, j\right) h\left(T_{3}, j\right) .
$$

Using $a_{i j}=a_{j i}$ we can rewrite these equations as follows:

$$
\begin{array}{r}
\operatorname{hom}_{k}\left(T^{\prime}(u), P_{n}\right)=\sum_{\substack{i \equiv k(2)\\
}} a_{i i} h\left(T_{1}, i\right) h\left(T_{3}, i\right) h\left(T_{2}, i\right) h\left(T_{4}, i\right) \\
+\sum_{\substack{i<j \\
i, j \equiv k(2)}} a_{i j}\left(h\left(T_{1}, i\right) h\left(T_{3}, i\right) h\left(T_{2}, j\right) h\left(T_{4}, j\right)\right. \\
\left.\quad+h\left(T_{1}, j\right) h\left(T_{3}, j\right) h\left(T_{2}, i\right) h\left(T_{4}, i\right)\right),
\end{array}
$$

and

$$
\begin{aligned}
\operatorname{hom}_{k}\left(T(u), P_{n}\right)= & \sum_{i \equiv k(2)} a_{i i} h\left(T_{1}, i\right) h\left(T_{3}, i\right) h\left(T_{2}, i\right) h\left(T_{4}, i\right) \\
& +\sum_{\substack{i<j \\
i, j \equiv k(2)}} a_{i j}\left(h\left(T_{1}, i\right) h\left(T_{4}, i\right) h\left(T_{2}, j\right) h\left(T_{3}, j\right)\right. \\
& \left.\quad+h\left(T_{1}, i\right) h\left(T_{4}, i\right) h\left(T_{2}, j\right) h\left(T_{3}, j\right)\right) .
\end{aligned}
$$

Copyright $@$ by SIAM. Unauthorized reproduction of this article is prohibited. 

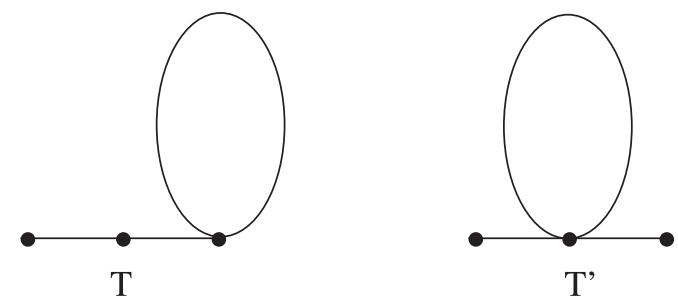

FIG. 6. Short-path shift.

Hence

$$
\begin{aligned}
& \operatorname{hom}_{k}\left(T^{\prime}(u), P_{n}\right)-\operatorname{hom}_{k}\left(T(u), P_{n}\right) \\
& \quad=\sum_{\substack{i<j \\
i, j \equiv k(2)}} a_{i j}\left(h\left(T_{1}, i\right) h\left(T_{2}, j\right)-h\left(T_{1}, j\right) h\left(T_{2}, i\right)\right)\left(h\left(T_{3}, i\right) h\left(T_{4}, j\right)-h\left(T_{3}, j\right) h\left(T_{4}, i\right)\right) .
\end{aligned}
$$

By the correlation inequalities, the signs of

$$
h\left(T_{1}, i\right) h\left(T_{2}, j\right)-h\left(T_{1}, j\right) h\left(T_{2}, i\right)
$$

and

$$
h\left(T_{3}, i\right) h\left(T_{4}, j\right)-h\left(T_{3}, j\right) h\left(T_{4}, i\right)
$$

only depend on the positions of $i$ and $j$, so they are the same. Hence

$$
\operatorname{hom}_{k}\left(T^{\prime}(u), P_{n}\right)-\operatorname{hom}_{k}\left(T(u), P_{n}\right) \geq 0,
$$

as desired.

The main problem with the LS-switch is that it preserves the sizes of the color classes of the tree (considered as a bipartite graph). So we need another transformation which can help us to compare trees with different color class sizes. For this reason we introduce the following transformation called short-path shift, which is actually a special case of the odd-KC-transformation (i.e., $k$ is odd in Figure 4 ).

DEFINITION 4.11 (Short-path shift). Let $T^{\prime}$ be obtained from the rooted tree $T_{1}(u)$ by attaching it to the middle vertex of a path on 3 vertices. Let $T$ be obtained from $T_{1}(u)$ by attaching it to an end vertex of a path on 3 vertices. Then we say that $T^{\prime}$ is obtained from $T$ by a short-path shift (see Figure 6).

LEMMA 4.12. Let $T^{\prime}$ be a short-path shift of $T$. Then for any odd $n \geq 5$, we have

$$
g\left(T^{\prime}, P_{n}\right) \geq g\left(T, P_{n}\right)
$$

Proof. Let $\left(a_{1}, a_{2}, \ldots, a_{n}\right)$ be the hom-vector of $T_{1}(u)$. Then the hom-vector of $T(u)$ is

$$
\left(2 a_{1}, 3 a_{2}, 4 a_{3}, 4 a_{4}, \ldots, 4 a_{n-2}, 3 a_{n-1}, 2 a_{n}\right)
$$

and the hom-vector of $T^{\prime}(u)$ is

$$
\left(a_{1}, 4 a_{2}, 4 a_{3}, 4 a_{4}, \ldots, 4 a_{n-2}, 4 a_{n-1}, a_{n}\right)
$$

Copyright (C) by SIAM. Unauthorized reproduction of this article is prohibited. 
Hence

$$
g\left(T^{\prime}, P_{n}\right)=\left(4 \sum_{i \equiv 0(2)} a_{i}\right)\left(-3 a_{1}-3 a_{n}+4 \sum_{i \equiv 1(2)} a_{i}\right)
$$

while

$$
g\left(T, P_{n}\right)=\left(-a_{2}-a_{n-2}+4 \sum_{i \equiv 0(2)} a_{i}\right)\left(-2 a_{1}-2 a_{n}+4 \sum_{i \equiv 1(2)} a_{i}\right) .
$$

Using the symmetry $a_{i}=a_{n+1-i}$ we get that

$$
g\left(T^{\prime}, P_{n}\right)-g\left(T, P_{n}\right)=8 \sum_{\substack{i \equiv 1(2) \\ 3 \leq i \leq n-2}}\left(a_{2} a_{i}-a_{1} a_{i+1}\right) .
$$

By the log-concavity of the hom-vector, all $a_{2} a_{i}-a_{1} a_{i+1} \geq 0$ for $i \equiv 1(\bmod 2)$. Hence

$$
g\left(T^{\prime}, P_{n}\right) \geq g\left(T, P_{n}\right)
$$

as desired.

Observe that both transformations decrease the Wiener index (sum of the distances of every pair of vertices in $T$ ) strictly: $W\left(T^{\prime}\right)<W(T)$ if $T^{\prime}$ is obtained from $T$ by an LS-switch or short-path shift and $T^{\prime}$ is not isomorphic to $T$.

Proof of Theorem 4.8. For $n=3$ we have $g\left(T, P_{3}\right)=2^{|T|}$ as we mentioned in Remark 4.7, so there is nothing to prove. Hence we can assume that $n \geq 5$. We shall use Lemmas 4.10 and 4.12 .

Let us consider the tree $T_{m}^{*}$ on $m$ vertices for which $g\left(T_{m}^{*}, P_{n}\right)$ is minimal and among these trees it has the largest Wiener index. We show that $T_{m}^{*}$ is $P_{m}$. Assume for contradiction that $T_{m}^{*}$ is not $P_{m}$. Let $v$ be an end vertex of a longest path of $T_{m}^{*}$. Clearly, $v$ is a leaf; let $u$ be its unique neighbor. We distinguish two cases.

If $\operatorname{deg}(u) \geq 3$, then $T_{m}^{*}$ can be decomposed into two branches, one of which is a star on at least 3 vertices. (Otherwise, $v$ cannot be an end vertex of a longest path.) Hence $u$ has another neighbor $w$ which is a leaf. In this case, $T_{m}^{*}$ is an image of a tree $\bar{T}$ by a short-path shift with respect to the path vuw. Hence $g\left(\bar{T}, P_{n}\right) \leq g\left(T_{m}^{*}, P_{n}\right)$ and $W(\bar{T})>W\left(T_{m}^{*}\right)$. This contradicts the choice of $T_{m}^{*}$.

If $\operatorname{deg}(u)=2$, then let $w$ be the closest vertex to $v$ having degree at least 3. (Such a $w$ must exist, because $T_{m}^{*}$ is not $P_{m}$.) Note that $d(v, w) \geq 2$. Let us decompose the tree $T_{m}^{*}$ into branches $P(v, w), T_{1}(w)$, and $T_{3}(w)$ at the vertex $w$, where $T_{1}(w)$ and $T_{3}(w)$ are nontrivial trees. If $d(v, w)$ is even, then $T_{m}^{*}$ is an image of a tree $\bar{T}$ by an LS-switch, where $T_{2}(v)=T_{4}(v)$ are one-vertex trees. If $d(v, w)$ is odd (consequently $d(u, w)$ is even), then $T_{m}^{*}$ is an image of a tree $\bar{T}$ by an LS-switch, where $T_{2}(u)$ is a one-vertex tree and $T_{4}(u)$ is the rooted tree on the vertex set $\{u, v\}$. In both cases $g\left(\bar{T}, P_{n}\right) \leq g\left(T_{m}^{*}, P_{n}\right)$ and $W(\bar{T}) \geq W\left(T_{m}^{*}\right)$. In the first case, the second inequality is strict contradicting the choice of $T_{m}^{*}$. In the second case, it may occur that $T_{3}(w)$ is also an edge implying that $T_{m}^{*}=\bar{T}$. By changing the role of $T_{2}(v)$ and $T_{4}(v)$, we can ensure that $T_{1}(w)$ is also an edge (otherwise we get the same contradiction as before). Hence in this case $T_{m}^{*}$ is a path with an edge attached on the second vertex. In this case we can realize that it is a short-path shift of a path at the vertex $w$. Hence we get a contradiction in this case too, which finishes the proof of the theorem.

To prove Theorem 1.1(ii), we will distinguish two cases according to the parity of $m$. 
4.1. Trees on even number of vertices. Theorem 1.1(ii): $n$ is an odd, $m$ is an even positive integer. Let $m$ be odd and $n$ be even. Then for any tree $T_{m}$ on $m$ vertices we have

$$
\operatorname{hom}\left(T_{m}, P_{n}\right) \geq \operatorname{hom}\left(P_{m}, P_{n}\right) .
$$

Proof. If $m$ is even, then $g\left(P_{m}, P_{n}\right)=\frac{1}{4} \operatorname{hom}\left(P_{m}, P_{n}\right)^{2}$ since $h_{A}=h_{B}=$ $\frac{1}{2} \operatorname{hom}\left(P_{m}, P_{n}\right)$. (The color classes of $P_{n}$ are not symmetric, but the color classes of $P_{m}$ are symmetric so it is equally likely which color class goes to which.) Hence by Theorem 4.8 we have

$$
\operatorname{hom}\left(T_{m}, P_{n}\right) \geq 2 g\left(T_{m}, P_{n}\right)^{1 / 2} \geq 2 g\left(P_{m}, P_{n}\right)^{1 / 2}=\operatorname{hom}\left(P_{m}, P_{n}\right) .
$$

If $m$ is odd, then we still need to work a bit.

4.2. Trees on odd number of vertices. From now on $n$ and $m$ are odd. Since $m$ is odd, it makes sense to speak about the large and small color classes of the tree considered as a bipartite graph. If $T_{m}$ is a tree on $m$ vertices, then $S$ will denote the color class of size at most $\frac{m-1}{2}$ and $L$ will denote the color class of size at least $\frac{m+1}{2}$. $S$ and $L$ stand for small and large. The notation $\operatorname{hom}_{0}\left(T(S), P_{n}\right)$ denotes $\operatorname{hom}_{0}\left(T(u), P_{n}\right)$, where $u \in S$. Hence it means that the small color class goes to the even-indexed vertices of the path. We can similarly define $\operatorname{hom}_{1}\left(T(S), P_{n}\right)$.

The following is a simple observation, which asserts that the small class of the path $P_{m}$ "likes" to go the small class of the path $P_{n}$.

Lemma 4.13. Let $m$ be odd. Then,

$$
\operatorname{hom}_{0}\left(P_{m}(S), P_{n}\right) \geq \operatorname{hom}_{1}\left(P_{m}(S), P_{n}\right) .
$$

Proof. Let $u$ be a leaf of $P_{m}$. Note that $u \in L$. Let $v$ be its neighbor, and let $\left(a_{1}, a_{2}, \ldots, a_{n}\right)$ be the hom-vector of $P_{m-1}(v)$. Then the hom-vector of $P_{m}(v)$ is $\left(a_{1}, 2 a_{2}, \ldots, 2 a_{n-1}, a_{n}\right)$. Note that

$$
\operatorname{hom}_{0}\left(P_{m}(S), P_{n}\right)=\operatorname{hom}_{0}\left(P_{m}(v), P_{n}\right)=2 \sum_{j \equiv 0(2)} a_{j},
$$

while

$$
\operatorname{hom}_{1}\left(P_{m}(S), P_{n}\right)=\operatorname{hom}_{1}\left(P_{m}(v), P_{n}\right)=-2 a_{1}+2 \sum_{j \equiv 1(2)} a_{j}
$$

Note that

$$
\sum_{j \equiv 0(2)} a_{j}=\sum_{j \equiv 1(2)} a_{j}
$$

since $P_{m-1}$ has an even number of vertices. Hence

$$
\operatorname{hom}_{0}\left(P_{m}(S), P_{n}\right) \geq \operatorname{hom}_{1}\left(P_{m}(S), P_{n}\right) .
$$

The following theorem is the main result of this part of the proof. It will imply the minimality of the path.

THEOREM 4.14. Let $m$ be odd. Then for any tree $T_{m}$ on $m$ vertices we have

$$
\operatorname{hom}_{0}\left(T_{m}(S), P_{n}\right) \geq \operatorname{hom}_{0}\left(P_{m}(S), P_{n}\right) \text {. }
$$

Copyright (C) by SIAM. Unauthorized reproduction of this article is prohibited. 
Before the proof of Theorem 4.14, we show how it can be applied to complete our proof of Theorem 1.1(ii).

Theorem 1.1(ii): $n, m$ are odd positive integers. Let $n$ and $m$ be odd. For any tree $T_{m}$ on $m$ vertices we have

$$
\operatorname{hom}\left(T_{m}, P_{n}\right) \geq \operatorname{hom}\left(P_{m}, P_{n}\right)
$$

Proof. We need to show that

$$
\operatorname{hom}\left(T_{m}, P_{n}\right) \geq \operatorname{hom}\left(P_{m}, P_{n}\right)
$$

for any tree $T_{m}$ on $m$ vertices.

From Theorem 4.8 we know that

$$
g\left(T_{m}, P_{n}\right) \geq g\left(P_{m}, P_{n}\right)
$$

In other words,

$$
\operatorname{hom}_{0}\left(T_{m}(S), P_{n}\right) \operatorname{hom}_{1}\left(T_{m}(S), P_{n}\right) \geq \operatorname{hom}_{0}\left(P_{m}(S), P_{n}\right) \operatorname{hom}_{1}\left(P_{m}(S), P_{n}\right) .
$$

By Theorem 4.14 and Lemma 4.13 we have

$$
\operatorname{hom}_{0}\left(T_{m}(S), P_{n}\right) \geq \operatorname{hom}_{0}\left(P_{m}(S), P_{n}\right) \geq \operatorname{hom}_{1}\left(P_{m}(S), P_{n}\right) .
$$

These inequalities together imply (4.1), which completes the proof of Theorem 1.1(ii).

4.3. Proof of Theorem 4.14. Now we start to prove Theorem 4.14. We need a few lemmas. The first one is trivial, but crucial.

Lemma 4.15. Let $T_{1}$ be a tree on $m$ vertices, and let $u \in L$ be a leaf. Let $v \in S$ be an arbitrary vertex of the small class. Let $T_{2}$ be a tree obtained from $T_{1}$ by deleting the vertex $u$ from $T_{1}$ and attaching a leaf $u^{\prime}$ to $v$. (So we simply move a leaf of the large class to another place, but we take care not to change the sizes of the color classes.) Then

$$
\operatorname{hom}_{0}\left(T_{1}(S), P_{n}\right)=\operatorname{hom}_{0}\left(T_{2}(S), P_{n}\right) .
$$

Proof. Let $T^{*}=T_{1}-u=T_{2}-u^{\prime}$. Note that

$$
\operatorname{hom}_{0}\left(T_{1}(S), P_{n}\right)=2 \operatorname{hom}_{0}\left(T^{*}(S), P_{n}\right)
$$

since any homomorphism of $T^{*}$ into $P_{n}$, where the small class goes to even-indexed vertices, can be extended into a similar homomorphism of $T_{1}$ in exactly two ways. Similarly,

$$
\operatorname{hom}_{0}\left(T_{2}(S), P_{n}\right)=2 \operatorname{hom}_{0}\left(T^{*}(S), P_{n}\right)
$$

Hence

$$
\operatorname{hom}_{0}\left(T_{1}(S), P_{n}\right)=\operatorname{hom}_{0}\left(T_{2}(S), P_{n}\right) .
$$

We will use the following transformation too.

DeFINITION 4.16 (claw-deletion). Let $T^{\prime}$ be a tree which contains a claw, three leaves attached to the same vertex. Let $T$ be obtained from $T^{\prime}$ by deleting the three 


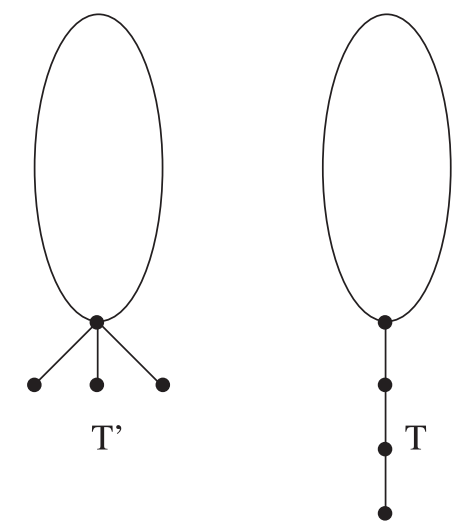

FIG. 7. Claw-deletion.

leaves and attaching a path of length 3 to the common neighbor of the leaves (see Figure 7). We call this transformation claw-deletion.

Note that the claw-deletion changes the sizes of the color classes. We have to be careful when we apply it, because it may occur that claw-deletion changes the small class into the large one.

We need the following property of the claw-deletion.

Lemma 4.17. Let $T^{\prime}$ be a tree on $m$ vertices with color classes $S^{\prime}$ and $L^{\prime}$. Assume that $\left|L^{\prime}\right|-\left|S^{\prime}\right| \geq 3$ and $T^{\prime}$ contains a claw. Let $T$ be obtained from $T^{\prime}$ by a clawdeletion, where we assume that the center $v$ of the claw is in the class $S^{\prime}$. Then

$$
\operatorname{hom}_{0}\left(T^{\prime}\left(S^{\prime}\right), P_{n}\right)>\operatorname{hom}_{0}\left(T(S), P_{n}\right) .
$$

Proof. Note that the condition $\left|L^{\prime}\right|-\left|S^{\prime}\right| \geq 3$ guarantees that the small class cannot become the large one in $T$. Let $u_{1}, u_{2}, u_{3}$ be the leaves of $T$, and let $v$ be their common neighbor. Let $T^{*}=T^{\prime}-\left\{u_{1}, u_{2}, u_{3}\right\}$. Let $\left(a_{1}, a_{2}, \ldots, a_{n}\right)$ be the hom-vector of $T^{*}(v)$. Then the hom-vector of $T^{\prime}(v)$ is

$$
\left(a_{1}, 8 a_{2}, \ldots, 8 a_{n-1}, a_{n}\right),
$$

and the hom-vector of $T(v)$ is

$$
\left(3 a_{1}, 6 a_{2}, 7 a_{3}, 8 a_{4}, \ldots, 6 a_{n-1}, 3 a_{n}\right)
$$

if $n \geq 7$. If $n=5$, then the hom-vector of $T^{\prime}(v)$ is $\left(a_{1}, 8 a_{2}, 8 a_{3}, 8 a_{4}, a_{5}\right)$, while the hom-vector of $T(v)$ is $\left(3 a_{1}, 6 a_{2}, 6 a_{3}, 6 a_{4}, 3 a_{5}\right)$. In both cases

$$
\operatorname{hom}_{0}\left(T^{\prime}\left(S^{\prime}\right), P_{n}\right)-\operatorname{hom}_{0}\left(T(S), P_{n}\right)=4 a_{2} .
$$

Our strategy will be the following. We transform a tree into a path by moving leaves and using the LS-switch and claw-deletion repeatedly. If we want to apply this last operation, we need to be sure that the condition $\left|L^{\prime}\right|-\left|S^{\prime}\right| \geq 3$ holds. The following lemma will be useful.

Lemma 4.18. Let $T$ be a tree with color classes $A$ and $B$. Assume that all leaves of $T$ belong to the color class $A$. Then $|A| \geq|B|$.

Proof. We prove the statement by induction on the number of vertices. If $T$ has at most 3 vertices, the claim is trivial. Assume that $T$ has $n$ vertices and we have 
proved the statement for trees on at most $n-1$ vertices. Let $u$ be a leaf of $T$, and let $v$ be its unique neighbor. Note that $u \in A$ and $v \in B$. Erase $u$ from $T$, and let $T^{*}$ be the obtained tree. If $v$ is a leaf in the obtained tree, then erase it as well and let us denote the resulting tree by $T^{* *}$, otherwise $T^{* *}=T^{*}$. (It may occur that $T^{* *}$ is the empty graph, but it is not a problem.) For $T^{* *}$ it is still true that one color class, $A^{* *}$, contains all leaves. Thus by induction $\left|A^{* *}\right| \geq\left|B^{* *}\right|$. Then

$$
|A|=\left|A^{* *}\right|+1 \geq\left|B^{* *}\right|+1 \geq|B|,
$$

as desired.

Remark 4.19. Let $T^{\prime}$ be a tree on $m$ vertices with color classes $L$ and $S$. Assume that $T^{\prime}$ contains a claw, and all leaves belong to $L$. Then $|L|-|S| \geq 3$. Indeed, if we delete two leaves from the claw, then it is still true for the resulting tree $T^{*}$ that all leaves belong to one color class, and it must be the larger one. Hence $\left|L^{*}\right| \geq\left|S^{*}\right|$, and since $m$ is odd, we have $\left|L^{*}\right| \geq\left|S^{*}\right|+1$. Thus for the original tree $T^{\prime}$ we have $|L|-|S| \geq 3$.

Proof of Theorem 4.14. Let $\mathcal{T}_{m}$ be the set of trees on $m$ vertices which minimizes $\operatorname{hom}_{0}\left(T(S), P_{n}\right)$. From $\mathcal{T}_{m}$ let us choose the tree $\bar{T}_{m}$ which has the smallest number of leaves and among these trees it has the largest Wiener index. We show that $\bar{T}_{m}$ must be $P_{m}$. Suppose for the sake of contradiction that $\bar{T}_{m} \neq P_{m}$.

First of all, $\bar{T}_{m}$ cannot be an image of an LS-switch, because if $\bar{T}_{m}$ can be obtained from a tree $F$ by an LS-switch, then by Lemma 4.10 we have

$$
\operatorname{hom}_{0}\left(F(S), P_{n}\right) \leq \operatorname{hom}_{0}\left(\bar{T}_{m}(S), P_{n}\right),
$$

and $F$ has at most as many leaves as $\bar{T}_{m}$, and $W(F)>W\left(\bar{T}_{m}\right)$. Hence it would contradict the choice of $\bar{T}_{m}$.

Let $S$ and $L$ be the small and large class of $\bar{T}_{m}$, respectively. Note that $L$ must contain a leaf by Lemma 4.18. We will distinguish two cases according to whether $S$ also contains a leaf or not.

Case 1. $S$ contains a leaf $v$. Let $x$ be the unique neighbor of $v$. First, we show that $x$ has degree at least 3 . If $\operatorname{deg}(x)=2$, then let $w$ be the closest vertex to $v$ having degree at least 3 . Such a $w$ must exist, because $\bar{T}_{m}$ is not $P_{m}$. Note that $d(v, w) \geq 2$. Let us decompose the tree $\bar{T}_{m}$ into branches $P(v, w), T_{1}(w)$, and $T_{3}(w)$ at the vertex $w$, where $T_{1}(w)$ and $T_{3}(w)$ are nontrivial trees. If $d(v, w)$ is even, then $\bar{T}_{m}$ is an image of a tree $F$ by an LS-switch, where $T_{2}(v)=T_{4}(v)$ are one-vertex trees. If $d(v, w)$ is odd (consequently $d(x, w)$ is even), then $\bar{T}_{m}$ is an image of a tree $F$ by an LS-switch, where $T_{2}(x)$ is a one-vertex tree and $T_{4}(x)$ is the rooted tree on the vertex set $\{x, v\}$. In the second case, it may occur that $T_{3}(w)$ is also an edge implying that $\bar{T}_{m}=F$. By changing the role of $T_{2}(v)$ and $T_{4}(v)$, we can ensure that $T_{1}(w)$ is also an edge (otherwise we get the same contradiction as before). Hence in this case $\bar{T}_{m}$ is a path with an edge attached on the second vertex. In this case we can realize that it is an LS-switch of a path since $m$ is odd. Hence we get a contradiction in this case too.

Now let $u \in L$ be a leaf. Let us delete it, and attach $u^{\prime}$ to $v$. This way we get a tree $T^{\prime}$. By Lemma 4.15 we know that

$$
\operatorname{hom}_{0}\left(\bar{T}_{m}(S), P_{n}\right)=\operatorname{hom}_{0}\left(T^{\prime}(S), P_{n}\right) .
$$

Furthermore, $T^{\prime}$ is an LS-switch (in fact, an even-KC-transformation) of a tree $F$. Indeed, let us decompose the tree $T^{\prime}$ at $x$ to $T_{1}(x), T_{2}(x)$, and the path $x v u^{\prime}$. Thus if we move $T_{2}(x)$ from $x$ to $u^{\prime}$, we get a tree $F$ for which

$$
\operatorname{hom}_{0}\left(F(S), P_{n}\right) \leq \operatorname{hom}_{0}\left(T^{\prime}(S), P_{n}\right) \text {. }
$$


Note that $u^{\prime}$ and $v$ are not a leaf in $F$ anymore. Possibly, the original neighbor of $u$ became a leaf in $F$, but still the number of leaves of $F$ is strictly less than the number of leaves of $\bar{T}_{m}$. This contradicts the choice of $\bar{T}_{m}$. Hence we are done in this case.

Case 2. $S$ contains no leaf. Hence all leaves belong to the class $L$. Let $u_{1} P u_{2}$ be the longest path of $\bar{T}_{m}$. As in the previous case, the unique neighbors $x_{1}$ and $x_{2}$ of $u_{1}$ and $u_{2}$, respectively, have degree at least 3 . We can assume that $x_{1} \neq x_{2}$, otherwise $\bar{T}_{m}$ is a star and it contains a claw and we can do claw-deletion, which strictly decreases $\operatorname{hom}_{0}\left(T(S), P_{n}\right)$. Since $x_{1}$ and $x_{2}$ have degree at least 3 , and $u_{1} P u_{2}$ was the longest path, the only possible way it can occur is that $x_{1}$ and $x_{2}$ have other neighbors $u_{3}$ and $u_{4}$, respectively, which are leaves. Now let us delete $u_{3}$ and add a new neighbor $u_{3}^{\prime}$ to $x_{2}$. Let $T^{\prime}$ be the obtained tree. Then by Lemma 4.15

$$
\operatorname{hom}_{0}\left(\bar{T}_{m}(S), P_{n}\right)=\operatorname{hom}_{0}\left(T^{\prime}(S), P_{n}\right) .
$$

On the other hand, it is still true that all leaves of $T^{\prime}$ belong to its large class. Moreover, it contains a claw: $\left\{x_{2}, u_{2}, u_{3}^{\prime}, u_{4}\right\}$. By Remark 4.19 the conditions of Lemma 4.17 are satisfied, and we can do a claw-deletion. Then we get a tree $F$ for which

$$
\operatorname{hom}_{0}\left(\bar{T}_{m}(S), P_{n}\right)=\operatorname{hom}_{0}\left(T^{\prime}(S), P_{n}\right)>\operatorname{hom}_{0}\left(F(S), P_{n}\right) .
$$

This contradicts the choice of $\bar{T}_{m}$.

Hence we get contradictions in all cases.

5. Final remarks, open problems. The main achievement of this paper is the inequality

$$
\operatorname{hom}\left(P_{m}, P_{n}\right) \leq \operatorname{hom}\left(T_{m}, P_{n}\right) \text { for any } m, n \text {. }
$$

Though this result is not unexpected, its proof turns out to be delicate, especially in the case when $m>n$. In view of Sidorenko's inequality (1.4), one may wonder if for any graph $G$

$$
\operatorname{hom}\left(P_{m}, G\right) \leq \operatorname{hom}\left(T_{m}, G\right)
$$

holds for any tree $T_{m}$ on $m$ vertices. This is not true even if one restricts $G$ to be a tree; see [7, Remark 4.13] for counterexamples.

There is an open problem left in Figure 2, which if true, would provide an alternative approach to [7, Theorem 1.8], which asserts the path has the fewest number of endomorphisms among all trees with fixed number of vertices.

Problem 5.1. Is it true that

$$
\operatorname{hom}\left(P_{n}, T_{n}\right) \leq \operatorname{hom}\left(T_{n}, T_{n}\right)
$$

for every tree $T_{n}$ on $n$ vertices?

Problem 5.2. Let $T_{m}$ be a tree on $m$ vertices with at least 4 leaves. Is it true that for any graph $G$ we have

$$
\operatorname{hom}\left(P_{m}, G\right) \leq \operatorname{hom}\left(T_{m}, G\right) ?
$$

If it is not true, then what is the smallest $f(m)$ such that if a tree $T_{m}$ on $m$ vertices has at least $f(m)$ leaves, then

$$
\operatorname{hom}\left(P_{m}, G\right) \leq \operatorname{hom}\left(T_{m}, G\right)
$$

for any graph $G$ ? 
The KC-transformation on trees seems quite natural, which has many applications in various extremal problems concerning trees. We are also interested in the topological aspects of the poset induced by the KC-transformation on trees. Let $P$ be a grated poset with $\hat{0}$ and $\hat{1}$. We say that the Möbius function (see [12, Chapter 3]) of $P$ alternates in sign if

$$
(-1)^{\ell(s, t)} \mu(s, t) \geq 0, \quad \text { for all } s \leq t \text { in } P .
$$

Let $\mathcal{K C}_{n}$ denote the graded poset on all trees with $n$ vertices that is induced by the KC-transformation. We have the following conjecture, which has been verified for $n \leq 8$.

COnjeCture 5.3. The Möbius function of $\mathcal{K C}_{n}$ alternates in sign for each $n \geq$ 1 .

It would be interesting to see if $\mathcal{K C}_{n}$ is $E L$-Shellable (see [13, section 3.2] for the definition), a property that implies alternating signs.

Acknowledgment. The authors wish to thank the anonymous referees for their valuable comments and suggestions.

\section{REFERENCES}

[1] B. BolLobÁs, Modern Graph Theory, Grad. Texts in Math. 184, Springer-Verlag, New York, 1998.

[2] B. Bollobás And M. Tyomkyn, Walks and paths in trees, J. Graph Theory, 70 (2012), pp. 5466.

[3] J.A. Bondy and U.S.R. Murty, Graph Theory, Springer-Verlag, New York, 2008.

[4] C. Borgs, J.T. Chayes, L. Lovász, V.T. Sós, and K. Vesztergombi, Counting graph homomorphisms, in Topics in Discrete Mathematics, M. Klazar, J. Kratochvil, M. Loebl, J. Matoušek, R. Thomas, and P. Valtr, eds., Springer, Berlin, 2006, pp. 315-371.

[5] P. CsikváRI, On a poset of trees, Combinatorica, 30 (2010), pp. 125-137.

[6] P. CsikváRI, On a poset of trees II, J. Graph Theory, 74 (2013), pp. 81-103.

[7] P. CsikváRi And Z. Lin, Graph homomorphisms between trees, Electron. J. Combin., 21 (2014), 4.9 .

[8] J. Cutler and J. Radcliffe, Extremal graphs for homomorphisms, J. Graph Theory, 67 (2011), pp. 261-284.

[9] J. Cutler AND J. RAdCliffe, Extremal graphs for homomorphisms II, J. Graph Theory, 76 (2014), pp. 42-59.

[10] J. EngBers And D. Galvin, H-colouring bipartite graphs, J. Combin. Theory Ser. B, 102 (2012), pp. 726-742.

[11] A. Sidorenko, A partially ordered set of functionals corresponding to graphs, Discrete Math., 131 (1994), pp. 263-277.

[12] R.P. Stanley, Enumerative Combinatorics, Vol. 1, 2nd ed., Cambridge University Press, Cambridge, 2011.

[13] M.L. WACHS, Poset topology: Tools and applications, Geometric Combinatorics, IAS/Park City Math. Ser. 13, AMS, Providence, RI, 2007, pp. 497-615.

Copyright (c) by SIAM. Unauthorized reproduction of this article is prohibited. 